\title{
GLOBAL SOLUTIONS OF THE CAUCHY PROBLEM FOR THE (CLASSICAL) COUPLED MAXWELLDIRAC AND OTHER NONLINEAR DIRAC EQUATIONS IN ONE SPACE DIMENSION
}

\begin{abstract}
V. DELGADO
ABSTRACT. The existence of global solutions is proved for the Maxwell-Dirac equations, for the Thirring model (Dirac equation with vector self-interaction), for the Klein-Gordon-Dirac equations and for two Dirac equations coupled through a vector-vector interaction (Federbusch model) in one space dimension. The proof is based on charge conservation, and depends on an "a priori" estimate of \|\|$_{\infty}$ for the Dirac field. This estimate is obtained only on the basis of algebraical properties of the nonlinear term, and allows us to simplify the proofs of global existence. We obtain it by computing $\partial_{0} j^{1}+\partial_{1} j^{0}$, with $\partial_{0} j^{0}+\partial_{1} j^{1}=0$ being the continuity equation which expresses charge conservation. We also prove global existence for the Thirring and Federbusch models coupled in standard form with the electromagnetic field.
\end{abstract}

1. Introduction. During the last years particular attention has been devoted to the study of global solutions to the Cauchy problem for certain physically interesting partial differential equations. Chadam [2] has proved global existence for Maxwell-Dirac equations in one space dimension, and subsequently extended the proof to Klein-Gordon-Dirac equations. Glassey [3] has studied the case of two Dirac equations coupled by means of a scalar and a Fermi interaction in one space dimension. He finds global existence, as well, whenever either the initial values or the coupling constant are small enough. Global existence has also been proved for the Thirring and Federbusch models by Salusti and Tesei [4]. Our concern in this paper is the study of global existence for the following equations:

(i) Coupled Maxwell-Dirac equations (M.D.):

$$
\begin{gathered}
\left(-i \gamma^{\mu} \partial_{\mu}+m\right) \psi=g v^{\mu} \gamma_{\mu} \psi, \\
\square v^{\mu}=\left(\partial_{0}^{2}-\partial_{1}^{2}\right) v^{\mu}=g \bar{\psi} \gamma^{\mu} \psi, \\
\partial^{\mu} v_{\mu}=0 ;
\end{gathered}
$$

(ii) Thirring model (T.M.):

Received by the editors March 8, 1977 and, in revised form, September 15, 1977.

AMS (MOS) subject classifications (1970). Primary 35A05.

Key words and phrases. Dirac equation, $\|\psi\|_{\infty}$ estimate, conservation law of charge, global existence. 


$$
\left(-i \gamma^{\mu} \partial_{\mu}+m\right) \psi=g\left(\bar{\psi} \gamma^{\mu} \psi\right) \gamma_{\mu} \psi
$$

(iii) Klein-Gordon-Dirac equations (K-G-D):

$$
\begin{gathered}
\left(-i \gamma^{\mu} \partial_{\mu}+m\right) \psi=g u \psi, \\
\square u+M^{2} u=g \bar{\psi} \psi ;
\end{gathered}
$$

(iv) Federbusch model (F.M.):

$$
\begin{aligned}
& \left(-i \gamma^{\mu} \partial_{\mu}+m_{1}\right) \psi=g\left(\bar{\varphi} \gamma^{\mu} \varphi\right) \gamma_{\mu} \psi \\
& \left(-i \gamma^{\mu} \partial_{\mu}+m_{2}\right) \varphi=g\left(\bar{\psi} \gamma^{\mu} \psi\right) \gamma_{\mu} \varphi
\end{aligned}
$$

(v) F. M. and T. M. coupled with the electromagnetic field.

We have chosen these equations, for their physical meaning, out of the set of those which allow us to obtain an "a priori" bound on \|\|$_{\infty}$ for the Dirac field.

$v^{\mu}$ are the components of the electromagnetic field (real vector), $\psi$ and $\varphi$ are Dirac spinor fields (in one space dimension spin space has two components). The scalar product in spin space is denoted by $\psi^{+} \psi, \psi^{+}$being the conjugate transpose of $\psi$. The adjoint spinor is denoted by $\bar{\psi}=\psi^{+} \gamma^{0} \cdot \gamma^{\mu}$ are operators in spin space which satisfy

$$
\gamma^{\mu} \gamma^{\nu}+\gamma^{\nu} \gamma^{\mu}=2 g^{\mu \nu} \quad\left(g^{00}=1, g^{11}=-1, g^{01}=g^{10}=0\right)
$$

and

$$
\gamma^{0 *}=\gamma^{0}, \quad \gamma^{1 *}=-\gamma^{1}
$$

All representations of the $\gamma^{\mu}$ are unitarily equivalent. $u$ is a real scalar field.

These equations are written in covariant form. Since we will also use their vector valued form, we define the matrices $\alpha$ and $\beta$ in this way:

they satisfy:

$$
\alpha=-\gamma^{0} \gamma^{1}, \quad \beta=-i \gamma^{0}
$$

$$
\alpha^{*}=\alpha, \quad \alpha^{2}=I, \quad \beta^{*}=-\beta, \quad \beta^{2}=-I, \quad \alpha \beta+\beta \alpha=0 .
$$

2. Mathematical formulation of the problem. Let us first introduce the following spaces [2]:

Definition. Let $M$ be the Hilbert space of square integrable functions $\left(\begin{array}{c}v \\ \dot{v}\end{array}\right)$ from $E^{1}$ with values in $\mathbf{R}^{2} \oplus \mathbf{R}^{2}$. Denote the diagonal operator $(-\Delta)^{1 / 2}$ by $B$. Then $M^{1 / 2}$ is defined to be $D(B) \oplus L^{2}$ endowed with the norm

$$
\left\|\left(\begin{array}{c}
v \\
\dot{v}
\end{array}\right)\right\|_{M_{1 / 2}}=\left\{\|B v\|_{2}^{2}+\|v\|_{2}^{2}+\|\dot{v}\|_{2}^{2}\right\}^{1 / 2}
$$

Definition. Let $D$ be the Hilbert space of spatially square integrable functions with values in spin space. Denote the diagonal operator $\left(m^{2} I-\right.$ $\Delta)^{1 / 2}$ by $A$. Then $D_{1}$ is defined to be $D(A) \subset D$ endowed with the norm

$$
\|\psi\|_{D_{1}}=\|A \psi\|_{2} .
$$

In fact, we will use $D_{1} \oplus M_{1 / 2}$ for Maxwell-Dirac, $D_{1}$ for the Thirring model, $D_{1} \oplus\left(D_{1} \oplus L^{2}\right)$ for Klein-Gordon-Dirac, and $D_{1} \oplus D_{1}$ for the 
Federbusch model. $D_{1}$ and $M_{1 / 2}$ are complete and equivalent, as Hilbert spaces, to a direct sum of Sobolev spaces $H_{1}$.

Now, we write the equations in the more convenient vector valued form. The equations read:

(i) Maxwell-Dirac:

$$
\begin{aligned}
\frac{d}{d t} \psi & =\left(\alpha \frac{\partial}{\partial x}+\beta m\right) \psi+g V \psi \\
\frac{d}{d t}\left(\begin{array}{c}
v \\
\dot{v}
\end{array}\right) & =\left(\begin{array}{ll}
0 & I \\
\Delta & 0
\end{array}\right)\left(\begin{array}{c}
v \\
\dot{v}
\end{array}\right)+g\left(\begin{array}{l}
0 \\
J
\end{array}\right)
\end{aligned}
$$

where

$$
v=\left(\begin{array}{c}
v_{0} \\
v_{1}
\end{array}\right), \quad V=i\left(v_{0}-v_{1} \alpha\right) \quad \text { and } \quad J=\left(\begin{array}{c}
j_{0} \\
j_{1}
\end{array}\right)=\left(\begin{array}{c}
\psi^{+} \psi \\
\psi^{+} \alpha \psi
\end{array}\right)
$$

(ii) Thirring model:

$$
\frac{d}{d t} \psi=\left(\alpha \frac{\partial}{\partial x}+\beta m\right) \psi+g U \psi,
$$

with $U=i\left(\psi^{+} \psi-\psi^{+} \alpha \psi \alpha\right)$;

(iii) Klein-Gordon-Dirac:

$$
\begin{aligned}
\frac{d}{d t} \psi & =\left(\alpha \frac{\partial}{\partial x}+\beta m\right) \psi-g u \beta \psi, \\
\frac{d}{d t}\left(\begin{array}{c}
u \\
\dot{u}
\end{array}\right) & =\left(\begin{array}{cc}
0 & 1 \\
-\left(m^{2}-\Delta\right) & 0
\end{array}\right) u+g\left(\begin{array}{c}
0 \\
i \psi+\beta \psi
\end{array}\right) ;
\end{aligned}
$$

(iv) Federbusch model:

$$
\frac{d}{d t}\left(\begin{array}{l}
\psi \\
\varphi
\end{array}\right)=\left[\left(\begin{array}{cc}
\alpha & 0 \\
0 & \alpha
\end{array}\right) \frac{\partial}{\partial x}+\left(\begin{array}{cc}
\beta m_{1} & 0 \\
0 & \beta m_{2}
\end{array}\right)\right]\left(\begin{array}{l}
\psi \\
\varphi
\end{array}\right)+\left(\begin{array}{cc}
U_{2} & 0 \\
0 & U_{1}
\end{array}\right)\left(\begin{array}{l}
\psi \\
\varphi
\end{array}\right),
$$

where

$$
U_{2}=i\left(\varphi^{+} \varphi-\varphi^{+} \alpha \varphi \alpha\right), \quad U_{1}=i\left(\psi^{+} \psi-\psi^{+} \alpha \psi \alpha\right) .
$$

If we now write them in integral form, with initial data $\psi_{0}, \varphi_{0}, u_{0}, \dot{u}_{0}, \ldots$, we get:

(i) Maxwell-Dirac:

$$
\begin{gathered}
\psi(t)=D\left(t-t_{0}\right) \psi_{0}-g \int_{t_{0}}^{t} D(t-s) V(s) \psi(s) d s \\
\left(\begin{array}{c}
v(t) \\
\dot{v}(t)
\end{array}\right)=M\left(t-t_{0}\right)\left(\begin{array}{c}
v_{0} \\
\dot{v}_{0}
\end{array}\right)-g \int_{t_{0}}^{t} M(t-s)\left(\begin{array}{c}
0 \\
J(s)
\end{array}\right) d s
\end{gathered}
$$

where

$$
D(t)=\exp \left(\alpha \frac{\partial}{\partial x}+\beta m\right) t, \quad M(t)=\left(\begin{array}{cc}
\cos t B & B^{-1} \sin t B \\
-B \sin t B & \cos t B
\end{array}\right)
$$


are the Dirac and Maxwell propagators, respectively.

(ii) Thirring model:

$$
\psi(t)=D\left(t-t_{0}\right) \psi_{0}-g \int_{t_{0}}^{t} D(t-s) U(s) \psi(s) d s,
$$

with

$$
D(t)=\exp \left(\alpha \frac{\partial}{\partial x}+\beta m\right) t
$$

(iii) Klein-Gordon-Dirac:

$$
\begin{aligned}
\psi(t) & =D\left(t-t_{0}\right) \psi_{0}+g \int_{t_{0}}^{t} D(t-s) u(s) \beta \psi(s) d s \\
\left(\begin{array}{c}
u(t) \\
\dot{u}(t)
\end{array}\right) & =K\left(t-t_{0}\right)\left(\begin{array}{c}
u_{0} \\
\dot{u}_{0}
\end{array}\right)-g \int_{t_{0}}^{t} K(t-s)\left(\begin{array}{c}
0 \\
i \psi^{+} \beta \psi
\end{array}\right), \\
K(t) & =\left(\begin{array}{cc}
\cos t A & A^{-1} \sin t A \\
-A \sin t A & \cos t A
\end{array}\right) ;
\end{aligned}
$$

(iv) Federbusch model:

with

$$
\begin{aligned}
\left(\begin{array}{l}
\psi(t) \\
\varphi(t)
\end{array}\right)= & C\left(t-t_{0}\right)\left(\begin{array}{l}
\psi_{0} \\
\varphi_{0}
\end{array}\right) \\
& -g \int_{t_{0}}^{t} C(t-s)\left(\begin{array}{cc}
U_{2}(s) & 0 \\
0 & U_{1}(s)
\end{array}\right)\left(\begin{array}{l}
\psi(s) \\
\varphi(s)
\end{array}\right) d s,
\end{aligned}
$$

$$
C(t)=\exp \left\{\left[\left(\begin{array}{ll}
\alpha & 0 \\
0 & \alpha
\end{array}\right) \frac{\partial}{\partial x}+\left(\begin{array}{cc}
\beta m_{1} & 0 \\
0 & \beta m_{2}
\end{array}\right)\right] t\right\}
$$

The local existence of solutions for (the integral form of) the Maxwell-Dirac and Klein-Gordon equations is obtained in [2] using the Segal fundamental theorem [1, Theorem 1]. In the same way, we can prove local existence for the Thirring and Federbusch models, provided that $D(t)$ and $C(t)$ are strongly continuous uniparametric unitary groups on $D_{1}$ and $D_{1} \oplus D_{1}$. The nonlinear terms are cubics and, hence, locally Lipschitz in $D_{1}$ and $D_{1} \oplus D_{1}$. (We use the Sobolev inequality $\|f\|_{\infty} \leqslant K\|f\|_{2}^{1 / 2}\|D f\|_{2}^{1 / 2}$.)

In order to prove global existence, we need to show that the norm of the solution remains finite for all $t>0$.

3. Main result. We begin the proof of global existence obtaining an "a priori" bound on $\|\psi\|_{\infty}$. Let us state and prove the following

THEOREM. Let $\psi$ represent a certain solution of the Maxwell-Dirac or Thirring model equations. Then $\|\psi\|_{\infty}<f, f$ being a function such that $f(t)<$ $\infty$ if $t<\infty$.

Proof.. If $\psi$ is a solution to our problem, it must, as well, satisfy the 
following equations:

$$
\frac{\partial}{\partial t} j^{0}+\frac{\partial}{\partial x} j^{1}=0, \quad j^{0}=\psi^{+} \psi, \quad j^{1}=-\psi^{+} \alpha \psi .
$$

This is nothing but the continuity equation which expresses charge conservation.

We now compute the "inverse" of this conservation law $\partial j^{1} / \partial t+\partial j^{0} / \partial x$; after some straightforward calculations we obtain

$$
\frac{\partial}{\partial t} j^{1}+\frac{\partial}{\partial x} j^{0}=-2 m \psi^{+} \alpha \beta \psi=2 m j^{5} .
$$

We must note that the algebraical properties of the nonlinear term are essential to get this result. In fact, for a scalar self-interaction, or two scalar coupled Dirac equations, this result is not true.

Charge conservation leads to $\|\psi\|_{2}=$ constant. (3a) and (3b) can be written as

$$
\frac{\partial}{\partial t}\left(\begin{array}{l}
j^{0} \\
j^{1}
\end{array}\right)+A \frac{\partial}{\partial x}\left(\begin{array}{l}
j^{0} \\
j^{1}
\end{array}\right)=2 m\left(\begin{array}{l}
0 \\
j^{5}
\end{array}\right) \text { with } A=\left(\begin{array}{ll}
0 & 1 \\
1 & 0
\end{array}\right) .
$$

$A$ is diagonalized by the unitary matrix $2^{-1 / 2}\left(\begin{array}{ll}1 & -1 \\ 1\end{array}\right)$.

The diagonalized system can be integrated on characteristics to yield

$$
\begin{aligned}
j^{0}(x, t)= & \frac{1}{2}\left(j_{0}^{0}(x-t)+j_{0}^{1}(x-t)+j_{0}^{0}(x+t)-j_{0}^{1}(x+t)\right) \\
& +m \int_{t_{0}}^{t}\left\{j^{5}(x-t+s, s)-j^{5}(x+t-s, s) d s\right. \\
j^{1}(x, t)= & \frac{1}{2}\left(j_{0}^{0}(x-t)+j_{0}^{1}(x-t)-j_{0}^{0}(x+t)+j_{0}^{1}(x+t)\right. \\
& +m \int_{t_{0}}^{t}\left\{j^{5}(x-t+s, s)+j^{5}(x+t-s, s)\right\} d s .
\end{aligned}
$$

Applying now \|\|$_{\infty}$ to both sides of the above expression, provided that $\left\|j^{5}\right\|_{\infty} \leqslant\left\|j^{0}\right\|_{\infty},\left\|j^{1}\right\|_{\infty} \leqslant\left\|j^{0}\right\|_{\infty}$, and using the Gronwall lemma, we obtain

$$
\left\|\left(\begin{array}{l}
j^{0} \\
j^{1}
\end{array}\right)\right\|_{\infty} \leqslant k_{1} e^{k_{2} t}
$$

This implies $\|\psi\|_{\infty}<f$, with $f$ under the conditions of the theorem, and $\psi$ being a solution to the M. D. or T. M. equations.

For the Federbusch model the equations which correspond to (3a), (3b) are

$$
\begin{gathered}
\frac{\partial}{\partial t} j^{0}+\frac{\partial}{\partial x} j^{1}=0, \quad j^{0}=\psi^{+} \psi, \quad j^{1}=-\psi^{+} \alpha \psi \\
\frac{\partial}{\partial t} k^{0}+\frac{\partial}{\partial x} k^{1}=0, \quad k^{0}=\varphi^{+} \varphi, \quad k^{1}=-\varphi^{+} \alpha \varphi \\
\frac{\partial}{\partial t} j^{1}+\frac{\partial}{\partial x} j^{0}=-2 m_{1} \psi^{+} \alpha \beta \psi=2 m_{1} j^{5} \\
\frac{\partial}{\partial t} k^{1}+\frac{\partial}{\partial x} k^{0}=-2 m_{2} \varphi^{+} \alpha \beta \varphi=2 m_{2} k^{5}
\end{gathered}
$$


In the same way we obtain a bound on $\left\|\left({ }_{\varphi}^{\psi}\right)\right\|_{\infty}$.

In the case of Klein-Gordon-Dirac, the identity corresponding to (3b) is

$$
\frac{\partial}{\partial x} \psi^{+} \psi-\frac{\partial}{\partial t} \psi^{+} \alpha \psi=-2(m-g u) \psi^{+} \alpha \beta \psi
$$

If we have an estimate on $\|u\|_{\infty}$ we can similarly obtain a bound on $\|\psi\|_{\infty}$. But it is known [2] that $u$ admits the representation

$$
\begin{aligned}
u(x, t)= & \frac{1}{2}\left\{u^{0}(x-t)+u^{0}(x+t)\right\} \\
& +\frac{1}{2} \int_{x-t}^{x+t} J_{0}\left(-M \sqrt{(x-\xi)^{2}-t^{2}}\right) \dot{u}^{0}(\xi) d \xi \\
& +\frac{1}{2} \int_{t_{0}}^{t}\left[\int_{x-(t-\tau)}^{x+(t-\tau)} J_{0}\left(-M \sqrt{(x-\xi)^{2}-(t-\tau)^{2}}\right) \bar{\psi} \psi(\xi, \tau) d \xi\right] d \tau
\end{aligned}
$$

Taking \|\|$_{\infty}$ and using the uniform boundedness of the zeroth order Bessel function $J_{0}$, we can obtain a bound on $\|u\|_{\infty},\|u\|_{\infty} \leqslant \operatorname{const}(1+t)[2$, Lemma 3.2]. Hence, $\|\psi\|_{\infty}<\infty \forall t<\infty$.

If the initial values of the Cauchy data possess enough derivatives in $L^{2}$, every solution of the integral equation is a solution of the differential equation, and continuity allows us to extend the bound on $\|\psi\|_{\infty}$ to the spaces of definition.

4. Global existence. We will perform the proofs in detail for Maxwell-Dirac and for the Thirring model. One can prove global existence for KleinGordon-Dirac following step by step the proof for Maxwell-Dirac, and for the Federbusch model, following the one for the Thirring model.

Theorem. A global solution to Maxwell-Dirac and to the Thirring model equations exists with

$$
\left(\psi_{0},\left(\begin{array}{c}
v_{0} \\
\dot{v}_{0}
\end{array}\right)\right) \in D_{1} \oplus M_{1 / 2}, \quad \psi \in D_{1} .
$$

Proof. We need to show that $\left\|\left(\psi,\left(\begin{array}{c}v \\ i\end{array}\right)\right)\right\|_{D_{1} \oplus M_{1 / 2}}$ and $\|\psi\|_{D_{1}}$ remain finite.

(a) Maxwell-Dirac: From (2g), taking \|\|$_{M_{1 / 2}}$, we have

$$
\begin{aligned}
\left\|\left(\begin{array}{c}
v(t) \\
\dot{v}(t)
\end{array}\right)\right\|_{M_{1 / 2}} & \leqslant\left\|\left(\begin{array}{c}
v_{0} \\
\dot{v}_{0}
\end{array}\right)\right\|_{M_{1 / 2}}+g \int_{t_{0}}^{t}\left\|M(t-s)\left(\begin{array}{c}
0 \\
J(s)
\end{array}\right)\right\|_{M_{1 / 2}} d s \\
& \leqslant\left\|\left(\begin{array}{c}
v_{0} \\
\dot{v}_{0}
\end{array}\right)\right\|_{M_{1 / 2}}+g \int_{t_{0}}^{t}\left\|\left(\begin{array}{c}
0 \\
J(s)
\end{array}\right)\right\|_{M_{1 / 2}} d s \\
& \leqslant\left\|\left(\begin{array}{c}
v_{0} \\
\dot{v}_{0}
\end{array}\right)\right\|_{M_{1 / 2}}+g \int_{t_{0}}^{t} \text { const }\|\psi\|_{\infty}\|\psi\|_{2} d s
\end{aligned}
$$

and this implies that $\left\|\left(\begin{array}{c}v(t) \\ \dot{v}(t)\end{array}\right)\right\|_{M_{1 / 2}}$ is finite, $\forall t$ finite. Use has been made of the 
fact that the propagator $M(t)$ is orthogonal and of the bounds on $\|\psi\|_{\infty}$. $\|\psi\|_{2} .\left\|\left(\begin{array}{c}v(t) \\ \dot{v}(t)\end{array}\right)\right\|_{M_{1 / 2}}$ finite $\forall t<\infty$ implies $\|v\|_{2},\|D v\|_{2},\|\dot{v}\|_{2}$ are finite, $\forall t$ finite.

Applying now the Sobolev inequality $\|f\|_{\infty} \leqslant K\|f\|_{2}^{1 / 2}\|D f\|_{2}^{1 / 2}$ we reach $\|v(t)\|_{\infty}$ finite $\forall t$ finite.

Let us now calculate $\|\psi\|_{D_{1}}$. From $(2 \mathrm{~g})$ we have

$$
\begin{aligned}
\|\psi(t)\|_{D_{1}} & \leqslant\left\|\psi_{0}\right\|_{D_{1}}+g \int_{t_{0}}^{t}\|D(t-s) V(s) \psi(s)\|_{D_{1}} d s \\
& \leqslant\left\|\psi_{0}\right\|_{D_{1}}+g \int_{t_{0}}^{t}\|V(s) \psi(s)\|_{D_{1}} d s
\end{aligned}
$$

since the Dirac propagator is unitary on $D_{1}$. The integrand can be estimated to yield

$$
\begin{aligned}
&\|\psi(t)\|_{D_{1}} \leqslant \text { const }+g \int_{t_{0}}^{t} C\left\{\|V(s) \psi(s)\|_{2}+\|D V(s) \psi(s)\|_{2}\right\} d s \\
& \leqslant \text { const }+ \text { const } g \int_{t_{0}}^{t}\left\{\|v(s)\|_{\infty}\|\psi(s)\|_{2}\right.+\|D v(s)\|_{2}\|\psi(s)\|_{\infty} \\
&\left.+\|v(s)\|_{\infty}\|D \psi(s)\|_{2}\right\} d s \\
& \leqslant a+b \int_{t_{0}}^{t}\left\{\|v(s)\|_{\infty}\|\psi(s)\|_{2}+\|D v(s)\|_{2}\|\psi(s)\|_{\infty}\right. \\
&\left.+\|v(s)\|_{\infty}\|\psi(s)\|_{D_{1}}\right\} d s .
\end{aligned}
$$

We have used

$$
\|D f\|_{2} \leqslant\|f\|_{D_{1}} \leqslant C\left\{\|f\|_{2}+\|D f\|_{2}\right\} .
$$

Now, with the help of the bounds on $\|\psi\|_{\infty},\|v\|_{\infty},\|v\|_{2}$ and $\|D v\|_{2},\|\psi\|_{2}$, and using the Gronwall lemma, we get

$$
\|\psi(t)\|_{D_{1}} \leqslant f(t), \quad f(t)<\infty \quad \forall t<\infty,
$$

and, therefore, global existence.

(b)Thirring model: From (2h) we get

$$
\begin{gathered}
\|\psi(t)\|_{D_{1}} \leqslant\left\|\psi_{0}\right\|_{D_{1}}+g \int_{t_{0}}^{t}\|D(t-s) U(s) \psi(s)\|_{D_{1}} d s \\
\leqslant\left\|\psi_{0}\right\|_{D_{1}}+g \int_{t_{0}}^{t}\|U(s) \psi(s)\|_{D_{1}} d s
\end{gathered}
$$

since the Dirac propagator is unitary on $D_{1}$.

Using $\|D f\|_{2} \leqslant\|f\|_{D_{1}} \leqslant C\left\{\|f\|_{2}+\|D f\|_{2}\right\}$ we can estimate the integrand to yield 


$$
\begin{aligned}
& \|\psi(t)\|_{D_{1}} \leqslant\left\|\psi_{0}\right\|_{D_{1}}+\text { const } \int_{t_{0}}^{t}\left\{\|U(s) \psi(s)\|_{2}+\|D U(s) \psi(s)\|_{2}\right\} d s \\
& \leqslant a+b \int_{t_{0}}^{t}\left\{\|U(s)\|_{\infty}\|\psi(s)\|_{2}+\|U(s)\|_{\infty}\|D \psi(s)\|_{2}\right. \\
& \left.+\|\psi(s)\|_{\infty}\|D U(s)\|_{2}\right\} d s \\
& \leqslant a+b \int_{t_{0}}^{t}\left\{\|\psi(s)\|_{\infty}^{2}\|\psi(s)\|_{2}+\|\psi(s)\|_{\infty}^{2}\|D \psi(s)\|_{2}+d\|\psi(s)\|_{\infty}^{2}\|D \psi(s)\|_{2}\right\} d s \\
& \leqslant a+e \int_{t_{0}}^{t}\left\{\|\psi(s)\|_{\infty}^{2}\|\psi\|_{D_{1}}\right\} d s,
\end{aligned}
$$

where we have also used the fact that $U(s)$ is quadratic in $\psi$.

Applying the bound on $\|\psi\|_{\infty}$ and the Gronwall lemma, we obtain

$$
\|\psi(t)\|_{D_{1}} \leqslant f(t), \quad f(t)<\infty \quad \forall t<\infty
$$

and global existence is proved.

The proof of global existence for the Klein-Gordon-Dirac equations is similar to the proof for Maxwell-Dirac, and for the Federbusch model, we follow the proof for the Thirring model.

5. Thirring and Federbusch models coupled with the Maxwell field. The equations are

$$
\begin{gathered}
\left(-i \gamma^{\mu} \partial_{\mu}+m\right) \psi=g_{1} v^{\mu} \gamma_{\mu} \psi+g_{2} \bar{\psi} \gamma^{\mu} \psi \gamma_{\mu} \psi, \\
\square v^{\mu}=g_{1} \bar{\psi} \gamma^{\mu} \psi, \quad \partial_{\mu} v^{\mu}=0
\end{gathered}
$$

for the Thirring model coupled with the electromagnetic field, and analogous ones for the Federbusch model.

The proofs of global existence are trivial, taking into account the proofs for Maxwell-Dirac and Thirring model case, and following them.

6. Concluding comments. The obtainment of the bound on $\|\psi\|_{\infty}$ is based only on algebraical properties of the nonlinear term. No condition about the size of the coupling constant or the initial values of the Cauchy problem is required.

Thanks are due to Professor A. Galindo and Professor J. M. Chadam. I want to acknowledge the referee of this paper for pointing out to me the extension of the proof to the Klein-Gordon-Dirac equations.

\section{REFERENCES}

1. I. E. Segal, Nonlinear semigroups, Ann. of Math. (2) 78 (1963), 339-364.

2. J. M. Chadam, The Cauchy problem for the (classical) coupled Maxwell-Dirac equations in one space dimension, J. Functional Analysis 13 (1973), 173-184.

3. R. T. Glassey, On one dimensional coupled equations, Alexander von Humboldt Institute, D8 Munchen 2, Thereseintra 39 (preprint).

4. E. Salusti and A. Tesei, On a semigroup approach to quantum field equations, Nuovo Cimento A2 (1971), 122-138.

Junta de Energía Nuclear, Ciudad Universitaria, Madrid-3, Spain 